\title{
Character Segmentation for License Plate Recognition by K-Means Algorithm
}

\author{
Lihong Zheng ${ }^{1}$ and Xiangjian $\mathrm{He}^{2}$ \\ ${ }^{1}$ School of Computing and Maths, Charles Sturt University, Australia \\ lzheng@csu.edu.au \\ ${ }^{2}$ Faculty of E\&IT, University of Technology, Sydney, Australia \\ xiangjian.he@uts.edu.au
}

\begin{abstract}
In this paper an improved K-means algorithm is presented to cut character out of the license plate images. Although there are many existing commercial LPR systems, with poor illumination conditions and moving vehicle the accuracy impaired. After examination and comparison of different image segmentation approaches, the K-means algorithm based method gave better image segmentation results. The K-means algorithm was modified by introducing automatic cluster number determination by filtering SIFT key points. After modification it efficiently detects the local maxima that represent different clusters in the image. The process is successful by getting a clean license plate image. While testing by the OCR software, the experimental results show a high accuracy of image segmentation and significantly higher recognition rate. The recognition rate increased from about $86.6 \%$ before our proposed process to about $94.03 \%$ after all unwanted non-character areas are removed. Hence, the overall recognition accuracy of LPR was improved.
\end{abstract}

Keywords: image segmentation, LPR, K-means algorithm.

\section{Introduction}

There are many problems with the License plate recognition (LPR) systems. In this paper we present a system based on K-means algorithm which efficiently improves the accuracy of character segmentation.

LPR is a system to identify a vehicle by recognizing the captured license plate image. It has been applied in numerous applications such as automatically identifying vehicles in a car park, and detecting and verifying stolen vehicles. There are many commercial LPRs around the world. Among these existing systems, there are two types of LPR. One uses commercial Optical Character Recognition (OCR) software to recognize the characters. The second kind uses learning based method to identify the characters. Both of them claim higher accuracy (99\%) under controlled conditions and the cameras are mounted in fixed locations without mobility. However, with poor illumination conditions and moving vehicle the accuracy impaired. When using an OCR for character recognition, it is crucial to correctly remove the license plate boundaries after the step of license plate detection. No matter which OCR is used, the 
recognition accuracy will be significantly reduced if the characters are not properly segmented. Therefore the characters in a license plate need to be highlighted and separated from the background area to improve the recognition accuracy.

Image segmentation is one of the key processes in LPR. It partitions the image into some constituted parts so that each constituted part contains one character and can be extracted for further processing. Image segmentation can be approached from two different perspectives: contour based or region based approaches [1-2]. Contour based method is a gradient-based segmentation method. It attempts to find the edges or boundaries directly from their high gradient magnitudes. Edge or boundary based approaches [3-4], and active contour based methods [5], are some good examples. On the other hand, in the region based methods, the segmentation is usually based on discontinuity and similarity of the gray level of the image or other features, such as color, texture, shape, etc. However, most of these methods are not working well in some cases where in the captured video data, the characters are connected each other or with the boundary. And moreover, boundaries are often shown in similar pattern to the characters and sometimes boundaries are broken. Therefore, the recognition rate is very low due to poor segmentation of characters.

In this paper, we first present an overview of the different approaches in image segmentation. We then proposed the modified K-means algorithm in Section 4.3. It is applied in a real time LPR system. It can identify the character areas in a car license plate image and remove non character areas of license plates correctly and efficiently. The novelty of the proposed method is that the parameters in this algorithm are determined automatically in Section 4.3.1 and the optimal character region is founded by a smart searching in Section 4.3.2. Later, the experimental results verify that the proposed method is successful in terms of improving the recognition accuracy.

\section{Review on Image Segmentation}

Among various types of methods on image segmentation, two different types of image segmentation methods are contour based methods, and region methods. In this section, an overview of these two major different approaches on image segmentation is presented. The advantages and disadvantages of each type are discussed.

Firstly, a contour based method attempts to identify edge pixels and then link them together to form the required boundaries. The edge or boundary approach [3] is a gradient-based segmentation method. It attempts to find the edges directly from their high gradient magnitudes. The edge approach is similar to the boundary approach, and also uses gradient information. It measures the rate of change in a function such as the image brightness function. However, edge/no edge decision is made locally and prematurely, therefore random edge segments are found everywhere in the image [3].

Snakes, an active contour model [5], start with some initial boundaries represented in the form of spline curves, and iteratively modify them by applying various shrinking or expansion operations. They are autonomous and self-adapting in their search for a minimal energy state. They are relatively insensitive to noise and other ambiguities in the images because the integral operator is an inherent noise filter. They can be used to track dynamic objects in temporal as well as the spatial dimensions. However, they can often get stuck in local minima states. Their accuracy 
is governed by the convergence criteria used in the energy minimization technique; higher accuracies require tighter convergence criteria and hence, longer computation times. Therefore, time consuming and information loss are their main pitfalls.

A region based technique divides an image into smaller parts and merges neighboring areas that have the same features. Adjacent areas are merged according to some criteria such as homogeneity or sharpness of region boundaries. In the region approach, each pixel is assigned to a particular object or region. A good example of region based segmentation is the graph-based method [7]. Graph-based methods model the image as a weighted, undirected graph. Usually a pixel or a group of pixels are associated with nodes and edge weights define the similarity between the neighborhood pixels. The graph is then separated according to a certain design criterion to model "good" clusters. The process of dividing a graph is a recursive bipartition until some termination criterion is met. Often, the termination criterion is based on the same cost function. Some popular algorithms of this category are normalized cuts [8], random walker [9], minimum cut [7], minimum spanning treebased segmentation [10], and ratio cut [11]. These researchers are seeking different ways to generalize and perform efficient iterated region based segmentation.

K-means algorithm [12] is one kind of region based clustering methods. It aims to partition $\mathrm{n}$ observations into $\mathrm{k}$ clusters in which each observation belongs to the cluster with the nearest mean. It is to find the centers of natural clusters in the data as well as in the iterative refinement approach. The details are discussed in Section 4.3.

In general, contour based technique relies on edge or boundaries detection. The need to connect together broken contour lines makes this technique prone to failure in the presence of blurring. Active contour based method is iterative searching and time consuming and information loss are its shortcoming. The region approach isolates objects resting on a contrasting background from the scene by using thresholds. Image was partitioned into smaller parts by merging the neighboring areas with the same features, such as color, text, homogeneity or sharpness. However, stringent restrictions on merging criteria create fragmentation; lenient ones overlook blurred boundaries and over merge [13]. After improvement K-means algorithm based method does efficiently segment the image into regions by knowing the optimal number of clusters.

\section{License Plate Detection}

As shown in [14], the basic idea of the detection algorithm was to use a variable scanning window moving around on the input vehicle image. The size of basic scanning window is set to be $48 \times 16$ and then scaled up to $300 \times 100$. At each position, the image area covered by the scanning window was classified using a pretrained classifier as either a license-plate area (a positive decision) or a non-licenseplate area (a negative decision). The classifier used in this algorithm was a significant extension of Viola and Jones' work [6] to license plate detection.

In our algorithm we construct a six-layer cascaded classifier to increase the detection speed, in which the first two layers are based on global features, Edge Density and Edge Density Variance defined in [14], and the last four layers are based on local Haar-like features. The classification process can be taken as a degenerate 
decision tree containing multi-layer classifiers as shown in Figure 1. A positive result from the first classifier triggers the evaluation of a second classifier. A positive result from the second classifier triggers a third classifier, and so on. A negative outcome at any layer leads to the immediate rejection of the image region (block). It is commonly seen that, for a given vehicle image, the majority of evaluated image regions are negative. Therefore, the cascaded classifier shown in Figure 1 attempts to reject as many negatives as possible at the earlier stages. As its consequence, this cascaded classifier leads to fast license plate detection.

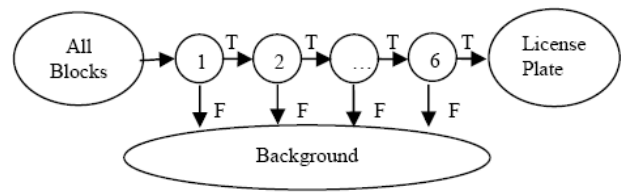

Fig. 1. Process of constructing a cascaded classifier [14]

To obtain the cascaded classifier which can make correct decisions, pre-classified positive samples (images containing license plates) and negative samples (images containing non-number-plates) are selected for training. In our experiments, all samples were obtained through manually labeling license plate areas in vehicle images captured under various conditions. The individual classifiers that together construct the cascaded classifier are trained independently.

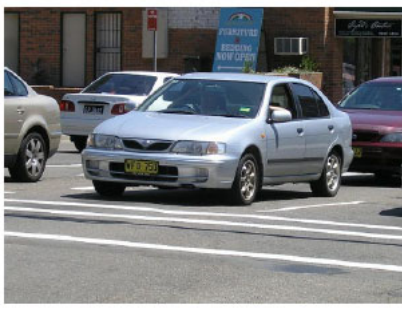

(a)

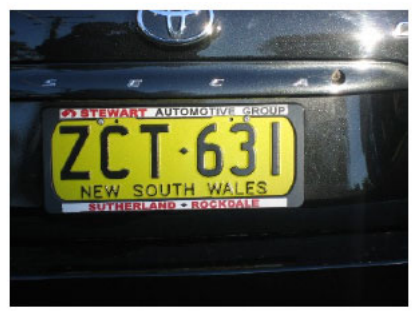

(b)

Fig. 2. Examples of small and large license plates

After the cascaded classifier has been trained and satisfies pre-defined classification accuracy, an input image is selected and a variable scanning window moves around the whole image space. To detect license plates of multiple sizes, the detection is carried out using multiple scales. Figure 2 gives an example that shows detectable license plates using our algorithm that have approximately the minimum size of $48 \times 16$ pixels (see Figure $2(\mathrm{a})$ ) and maximum size of $300 \times 100$ pixels (see Figure 2(b)). In both cases, the vehicle images have $512 \times 384$ pixels. 


\section{Character Segmentation of License Plates}

Once the license plate area is found, the following step is to find the characters contained in the license plate. The procedures of character segmentation include three different tasks. The first one is character height estimation. The upper and lower boundaries of character are located and used to obtain the character height. Then, we move to estimate character width. The final step is to cut character segments based on K-means algorithm.

\subsection{Character Height Estimation of License Plates}

This step contains three parts: colour reverse, vertical edge detection and horizontal projection histogram

\subsubsection{Colour Reverse}

The license plates in the New South Wales (NSW) State, Australia have many different formats, colours, and alignments. For instance, white in black, black in white, black in yellow, etc. are commonly used colour combinations. Colour reserve step is necessary before we assign right colour (i.e., black) to the characters of a license plate and hence obtain a correct binary image of the license plate. It makes the colour of the characters on a license plate be black. It is done based on a statistical analysis of edges. Given the located image, we pick $l$ horizontal symmetrical lines on the license plate image. The colour index $\mathrm{C}_{\mathrm{I}}$ is calculated as the average amount of the cross points (where pixel value changes between black and white) along each line in horizontal direction. Cross point number is increased by one if there is a foreground point.

$$
C_{I}=\frac{1}{l} \sum_{j} \sum_{i} F(i, j)
$$

where $\mathrm{j}$ is from 1 to $l$ and $i$ is from 1 to $N$, and $F(i, j)$ is 1 if it is foreground point at location $(i, j)$. If $C_{I}$ value is over a statistical selected threshold, the candidate image is labeled as an image to be converted. Otherwise keep the original value for next step.

Therefore, all candidate license plates will look as black-in-white, and the consistency of license plate colour combination is made.

\subsubsection{Vertical Edge Detection}

Once a rectangular area of the license plate is detected and located, a simple area enlargement computation is applied so that the enlarged area will fully contain the license plate. Figure 3 shows some examples of final rectangular areas of the license plate images.

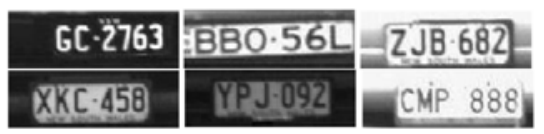

Fig. 3. Images Samples of Located License plates 
Note that the located area shows stronger connectivity in vertical direction than horizontal direction, we perform vertical edge detection on the license plate images as shown in Figure 4 through the computation of horizontal gradient. At each pixel, use

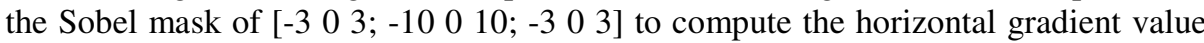
[15]. Then, use the Otsu [16] method for binarization to obtain the vertical maps. The edge pixels are represented using white pixels and other pixels using black pixels. Figure 4(a) shows the vertical edge pixels of the sample images.

\subsubsection{Horizontal Projection Histogram}

Although projection histogram is not a new concept, it is used here to find the upper and lower bounds of a license plate after the vertical edge are obtained. We perform a horizontal projection to find the top and bottom position of characters. When all values of histogram bins along all lines in the horizontal direction are computed, the horizontal projection histogram is obtained. The mean value of the histogram is then used as a threshold to figure out where the upper bound and lower bound are. The horizontal projection histograms of the three sample images are displayed in Figure 4(b). Finally, the distance between upper and lower boundaries is recorded as the height value of characters.

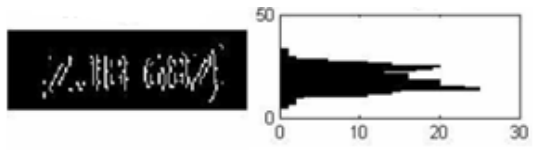

(a)

(b)

Fig. 4. (a) Vertical edge maps of images in Figure 3, (b) Horizontal projection histogram

\subsection{Character Width Estimation of License Plates}

The middle area between the upper and lower bounds is recorded and considered for character segmentation on the license plate. Image binarization and vertical projection are two steps for segmentation here. Each segment here may contain one or two characters. Note that we use segmentation results to estimate the width of the characters on the license plate for the following processes. Each segment does not need to be accurate to contain exactly one character.

Image binarization highlights the pixels of interest and suppresses the background pixels. Otsu's method [16] is used to optimally classify all pixels with values above this threshold as white ( 255 grey value), and all other pixels as black ( 0 grey value). Figure 5 shows the results of binarization on the images in Figure 3 after cutting the upper and lower boundaries of the license plates.

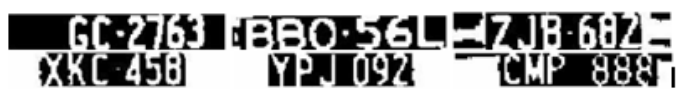

Fig. 5. Binarized images of the images in Figure 3 with upper and lower bounds removed 
Similarly, we perform a vertical histogram projection to find the gaps between characters on a license plate. Each license plate is separated into blocks horizontally by the zero points in the projection histogram. Figure 6 shows the vertical projection of three images in Figure 5 and the segments (or blocks) of the images.

To estimate the width of a character after the segmentation process above, we take into account only widths of all blocks except the two smallest blocks and the two biggest ones. The averaged widths of these blocks are used as the estimated width of characters. The estimated height and width of characters will be used in the following step for character extraction.

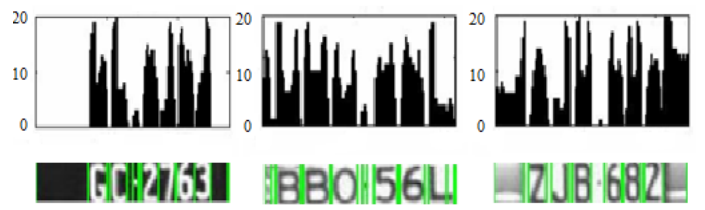

Fig. 6. Vertical projection of the images in Figure 5 and character segments

\subsection{K-Means Algorithm Based Accurate Character Segmentation}

\subsubsection{K-Means Algorithm}

The K-means [12] algorithm was invented in 1956. It is an iterative technique that is used to partition an image into $K$ clusters. The basic idea is as follows:

1) Pick $K$ cluster centers randomly,

2) Assign each pixel in the image to the nearest cluster based upon the similarity parameter such as Euclidean distance of intensity,

3) Recalculate the cluster centers for the new clusters by averaging all of the pixels in the cluster,

4) Repeat steps 2 and 3 until convergence is reached (e.g. the pixels no longer switch clusters).

K-Means algorithm typically converges to a solution very quickly as opposed to other clustering algorithms. But it may not return the optimal solution. A drawback of this algorithm is that the quality of the solution depends largely on the initial set of clusters and the value of $K$. An inappropriate choice of $K$ may yield poor results. The optimal $K$ value is identified automatically by SIFT based method described in the following section. The experimental results are shown in Figure 7 below.

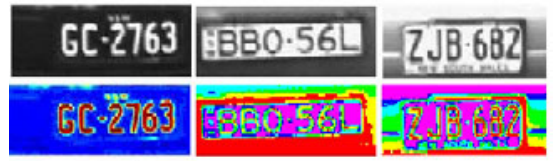

Fig. 7. Segmented areas in different colors of license plate sample images 


\subsubsection{Selection of $K$ by SIFT Algorithm}

Scale Invariant Feature Transform (SIFT) algorithm developed by Lowe David [17], is currently one of the best ways to find the invariant features. The SIFT features are proven that to be invariant to image scale and rotation, and are shown to provide robust matching across a substantial range of affine distortion, change in 3D viewpoint, addition of noise, and change in illumination. SIFT combines a scale invariant region detector using the Difference of Gaussian (DoG) and a descriptor based on gradient distribution in the detected regions. Firstly, local peaks (termed key-points) in a series of difference-of-Gaussian (DoG) images are found. Then, candidate key-points are located based on the measures of their stability. After filtering of SIFT key points make a good auto determination of cluster $K$ value for Kmeans algorithm. Figure 8 below show the examples where SIFT key points are.

Once these extremely discriminative SIFT features are obtained, it is important to have a prior that obtained in previous steps. After filtering some key points leading to more clusters, the optimal cluster $K$ value of the K-means algorithm turn out depend on the choice of prior knowledge such as character bounds.

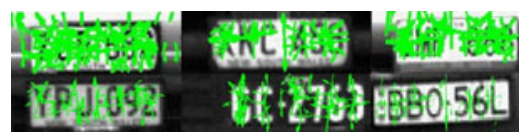

Fig. 8. Key points identified by SIFT algorithm

\section{Experiential Results and Comparison}

To test the recognition algorithm, we apply the proposed algorithm on 587 license plate images with 3502 characters located by the license plate detection step. All characters are correctly segmented. The false positives (i.e., non-character areas that are segmented) are mainly due to the false detection of license plates. Only 7 out of total 594 license plate detected regions are non-license plates. So our correct segmentation rate is still very high and is about $98.82 \%$ even taking into account the rate of wrongly detected license plate regions. The binary enlarged license plate images are obtained by our proposed method and are sent to the OCR software (Tesseract) for recognition. With slight modification of the OCR code, all segmented characters on the license plates are recognized correctly. A performance comparison with various methods is shown in Table I. Our license plate detection rate is $96.4 \%$.

Table 1. Performance Comparison

\begin{tabular}{lll}
\hline References & Detection Accuracy & $\begin{array}{l}\text { Segmentation/Recognition } \\
\text { Accuracy }\end{array}$ \\
\hline$[18]$ & $93.2 \%$ & $95 \%$ \\
{$[19]$} & $97.1 \%$ & $96.4 \%$ \\
Proposed & $96.4 \%$ & $98.82 \%$ \\
\hline
\end{tabular}


Furthermore, an empirical evaluation of the proposed segmentation method(s) and other segmentation methods such as traditional CCA and active contour (AC) based method [5] is presented.

Firstly, traditional CCA uses the connectivity between a pixel and its neighbors to label the pixel and merge into same group. Given an image, CCA assigns labels to a pixel such that adjacent pixels of the same features are assigned the same label. The image is copied into a small or large array. It does multiple scans to label the pixels as belonging to one of many different groups. However, if characters are connected each other, it is hard to separate them individually. Secondly, active contour based approach starts with some initial boundaries and iteratively modify them by applying some shrinking or expansion operations. It gets stuck in local minima states due to overlook minute features in the process of minimizing the energy over the entire path of their contours. It is a time consuming method. It takes more than eleven times of the time that CCA takes and ten times of the time that our approach uses. As shown in Table II, our approach found the best interested area of clear characters finally.

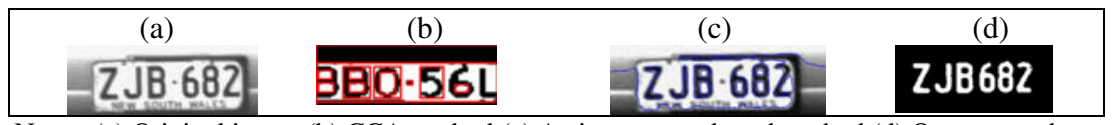

Notes: (a) Original image (b) CCA method (c) Active contour based method (d) Our approach

Fig. 9. Comparison of original license plate with images developed using other approaches

Table 2. Comparison of experimental results with images developed using three alternative approaches

\begin{tabular}{l|l|l|l}
\hline \multirow{2}{*}{\multicolumn{1}{c|}{ Accuracy rate of LPR }} & \multicolumn{3}{|c}{ Methods } \\
\cline { 2 - 4 } & $\boldsymbol{C C A}$ & $\boldsymbol{A C}$ & Ours \\
\hline Detection rate of license plates & $96.4 \%$ & $96.4 \%$ & $96.4 \%$ \\
Accuracy of character segmentation & $91.0 \%$ & $76.1 \%$ & $98.82 \%$ \\
Average time of segmentation (s) & 0.154 & 2.21 & 0.204 \\
Character recognition rate & $98.7 \%$ & $98.7 \%$ & $98.7 \%$ \\
Overall recognition rate & $86.6 \%$ & $71.9 \%$ & $94.03 \%$ \\
\hline
\end{tabular}

\section{Conclusions}

In this paper, we have constructed a cascaded classifier consisting of 6 layers for license plate detection using both global edge features and local Harr-like features. The classifiers on the first two layers exclude more than $80 \%$ non-plate regions from further training or testing and hence greatly increase the detection speed in the next four layers. The classifiers on the next four layers are based on local Haar-like features. With a small number of features, we can obtain very high detection rate with very low false positive rate even when the license plate detection algorithm is used under various complex environments. A real-time detection speed is achieved.

Moreover, we have proposed a method to segment the characters of car license plates. This is a crucial work after the detection of license plates and before the use of OCRs for character recognition. The process is successful through various techniques including image binarization, vertical edge detection, horizontal and vertical image projections, and modified K-means segmentation algorithm. Various well-known 
techniques are applied to come out with the innovative algorithm in this paper. The Tesseract OCR software was used to test our results. The experimental results show a significantly higher recognition rate $94.03 \%$ after character is segmented. Hence, the overall recognition accuracy has been improved.

\section{References}

1. Shapiro, L.G., Stockman, G.C.: Computer Vision. Prentice Hall, Englewood Cliffs (2002)

2. Achanta, R., Estrada, F., Wils, P., Süsstrunk, S.: Salient Region Detection and Segmentation. In: Gasteratos, A., Vincze, M., Tsotsos, J.K. (eds.) ICVS 2008. LNCS, vol. 5008, pp. 66-75. Springer, Heidelberg (2008)

3. Malik, J., Belongie, S., Shi, J., Leung, T.: Contour and Texture Analysis for Image Segmentation. International Journal of Computer Vision 43(1), 7-27 (2001)

4. Ko, B.C., Nam, J.Y.: Object-of-interest Image Segmentation based on Human Attention and Semantic Region Clustering. Journal of Optical Society of America A 23(10), 2462 2470 (2006)

5. Chan, T.F., Vese, L.A.: Active Contours without Edges. IEEE Transactions on Image Processing 10(2), 266-277 (2001)

6. Viola, P., Jones, M.J.: Robust Real-Time Face Detection. International Journal of Computer Vision 57(2), 137-154 (2004)

7. Shi, J., Malik, J.: Normalized Cuts and Image Segmentation. IEEE Transactions on Pattern Analysis and Machine Intelligence 22(8), 888-905 (2000)

8. Jermyn, I.H., Ishikawa, H.: Globally Optimal Regions and Boundaries as Minimum Ratio Cycles. IEEE Transactions on Pattern Analysis and Machine Intelligence 23(10), 1075$1088(2001)$

9. Sarkar, S., Soundararajan, P.: Supervised Learning of Large Perceptual Organization: Graph Spectral Partitioning and Learning Automata. IEEE Transactions on Pattern Analysis and Machine Intelligence 22(5), 504-525 (2000)

10. Soundararajan, P., Sarkar, S.: Analysis of Mincut, Average cut, and Normalized Cut Measures. In: Third Workshop Perceptual Organization in Computer Vision (2001)

11. Wang, S., Siskind, J.M.: Image Segmentation with Ratio Cut. IEEE Transactions on Pattern Analysis and Machine Intelligence 25, 675-690 (2003)

12. Kanungo, T., et al.: An Efficient K-means Clustering Algorithm: Analysis and Implementation. IEEE Transactions on Pattern Analysis and Machine Intelligence 24, 881-892 (2002)

13. Chazelle, B.: Application challenges to computational geometry: CG Impact Task Force Report. Technical Report TR-521-96, Princeton University (1996)

14. Zhang, H., Jia, W., He, X., Wu, Q.: Learning-based License Plate Detection in Vehicle Image Database. International Journal of Intelligent Information and Database Systems (IJIIDS), Inderscience 1(2), 228-243 (2007); ISSN: 1751-5858

15. http://www.pages.drexel . edu/ weg2 2 / edge.html

16. Otsu, N.: A Threshold Selection Method from Gray-level Histograms. IEEE Transactions on Systems, Man, and Cybernetics 9, 62-66 (1979)

17. Lowe, D.G.: Distinctive Image Features from Scale-Invariant Keypoints. International Journal of Computer Vision 60(2), 91-110 (2004)

18. Wu, C., et al.: A Macao License Plate Recognition System. In: IEEE Proceedings of the Fourth International Conference on Machine Learning and Cybernetics, Guangzhou, vol. 7, pp. 4506-4510 (2005)

19. Guo, J.M., et al.: License Plate Localization and Character Segmentation with Feedback Self-learning and Hybrid-binarization Techniques. In: IEEE Region 10 Conference, pp. 1-4 (2007) 